УДК 616-006.66

\title{
МЕДИЦИНСКИЕ НАУКИ
}

\section{РЕЗИДУАЛЬНЫЙ РАК МОЛОЧНОЙ ЖЕЛЕЗЫ}

${ }^{1}$ Ерещенко С.С., 1 Дашян Г.А., ${ }^{1,2}$ Криворотько П.В., ${ }^{1}$ Иванова О.А., ${ }^{1}$ Табагуа Т.Т., ${ }^{1}$ Губайдуллина М.А., ${ }^{1}$ Аполлонова В.С., ${ }^{2}$ Даниелян Д.А., ${ }^{3}$ Прокопенко А.Д., ${ }^{1}$ Семиглазов В.Ф. ${ }^{\prime}$ ФББУ «НМИЦ онкологии им. Н.Н. Петрова» Минздрава России, Санкт-Петербург;

${ }^{2}$ ФГБОУ ВО «Северо-Западный государственный медицинский университет им. И.И. Мечникова» Минздрава России, Санкт-Петербург;

${ }^{3}$ ФГБОУ ВО «Первый Санкт-Петербургский государственный медицинский университет им. акад. И.П. Павлова» Минздрава России, Санкт-Петербург, e-mail:dr.ereschenko@gmail.com

В исследовании Symmans и соавт. (2017) была поставлена цель определить долгосрочный прогноз при каждом фенотипическом подтипе РМЖ в зависимости от резидуальной (остаточной) опухоли (RCB) после одной неоадъювантной химиотерапии или ее комбинации с анти HER2-таргетной терапией. Авторы провели патоморфологический обзор измерений и оценок индекса RCB (residual cancer burden), в котором полный патоморфологический ответ обозначается как $\mathrm{RCB}=0$; а резидуальное заболевание категоризировалось в три предопределенных класса индекса RCB (RCB-I, RCB-II, RCB-III и постнеоадъювантную стадию ур остаточной опухоли). Пациенты наблюдались проспективно для анализа выживаемости. Фенотипические подтипы определялись по гормонально-рецепторному и HER2 статусу при первичном диагнозе и классифицировались как ER-позитивный/ HER2-негативный, HER2-позитивный (ER-негативный/ HER2-позитивный или ER-позитивный/HER2-позитивный), и трижды рецептор-негативный. Безрецидивная выживаемость устанавливалась с помощью анализа по Каплану - Майеру и сравнивались с помощью логрангового теста. Средний период наблюдения в пяти когортах (T/FAC-1 [n = 219], T/FAC-2 [n = 262], T/FAC-3 [n = 342$]$, FAC $[\mathrm{n}=132]$, и H+T/FEC [n = 203]) равнялся 13,5; 9,1; 6,8; 16,4 и 7,1 годам соответственно. Индекс RCB являлся прогностическим в пределах каждого фенотипического подтипа, независимо от других клинико-патоморфологических вариабельностей. RCB классы стратифицировали прогностический общий риск в пределах каждого фенотипического подтипа и в пределах категории ур-стадий установлены показатели 10-летней безрецидивной выживаемости в четырех RCB-классах (патоморфологический полный ответ, RCB-I, RCB-II, RCB-III) равнялись сответственно 86, 81, 55 и $23 \%$ для трижды-рецептор негативных опухолей; 83, 97, 74 и $52 \%$ для ER-позитивных/ HER2-негативных опухолей в комбинированных когортах T/FAC; и 95, 77, 47 и $21 \%$ в когорте Н+T/FEC. В целом индекс RCB оказался прогностическим для долгосрочной выживаемости после неоадъювантной химиотерапии во всех трех фенотипических РМЖ.

Ключевые слова: рак молочной железы, неоадювантная химиотерапия, резидуальный рак

\section{RESIDUAL BREAST CANCER}

${ }^{1}$ Erechshenko S.S., ${ }^{1}$ Dashyan G.A., ${ }^{1,2}$ Krivorotko P.V., ${ }^{1}$ Ivanova O.A., ${ }^{1}$ Tabagua T.T., ${ }^{1}$ Gubaydullina M.A., ${ }^{1}$ Apollonova V.S., ${ }^{2}$ Danielyan D.A., ${ }^{3}$ Prokopenko A.D., ${ }^{1}$ Semiglazov V.F. ${ }^{I}$ N.N. Petrov National Medical Research Center of Oncology, Ministry of Health of Russia, Saint Petersburg;

${ }^{2}$ North-Western State Medical University named after I.I. Mechnikov, Ministry of Health of Russia, Saint Petersburg;

${ }^{3}$ Acad. I.P. Pavlov First Saint Petersburg State Medical University, Saint Petersburg, e-mail:dr.ereschenko@gmail.com

In the study, Symmans et al. (2017), the goal was to determine the long-term prognosis for each phenotypic subtype of breast cancer as a function of residual (RCB) after one neoadjuvant chemotherapy or a combination with anti-HER2 therapy. The authors carried out a pathomorphological survey of the measurements and estimates of the RCB (residual cancer burden) index, in which the complete pathomorphological response is denoted as $\mathrm{RCB}=0$; and residual disease was categorized into three predetermined classes of the RCB index (RCB-I, RCB-II, RCB-III and post-neoadjuvant yp of the residual tumor). Patients were prospected for survival analysis. The mean observation period in five cohorts (T/ FAC-1 $[\mathrm{n}=219], \mathrm{T} /$ FAC-2 [ $\mathrm{n}=262]$, T / FAC-3 [ $\mathrm{n}=342]$, FAC $[\mathrm{n}=132]$, and H + T / FEC $[\mathrm{n}=203])$ was 13.5, 9.1, $6.8,16.4$, and 7.1 years, respectively. The RCB index was prognostic within each phenotypic subtype, regardless of other clinico-pathomorphological variability. RCB classes stratified the prognostic overall risk within each pfenotypic subtype, and 10-year disease-free survival in four RCB classes (pathological complete response, RCB-I, RCB-II, RCB-III) was established within the category of yp stages. $\%, 81 \%, 55 \%$ and $23 \%$ for the triple-receptor negative tumors; $83 \%, 97 \%, 74 \%$, and $52 \%$ for ER-positive / HER2-negative tumors in combined T / FAC cohorts; and $95 \%$, $77 \%, 47 \%$ and $21 \%$ in the $\mathrm{H}+\mathrm{T} / \mathrm{FEC}$ cohort. Overall, the RCB index was prognostic for long-term survival after neoadjuvant chemotherapy in all three phenotypic breast cancer.

Keywords: breast cancer, neoadjuvant chemotherapy, residual cancer

Механизм FDA для ускоренного одобрения химиотерапевтического лечения раннего (операбельного) РМЖ высокого риска базируется на увеличении частоты полного патоморфологического регресса опухоли (pCR) после неоадъювантного лечения в рандомизированном клиническом испытании [1-3]. Хотя этот подход демон- 
стрирует надежность в прогностической значимости pCR, все еще остается неясным, какова величина прогностического отличия может оказаться от улучшения частоты pCR [3-5]. Прогностическое отличие может также зависеть от распределения распространенности резидуального заболеваемости в каждом лечебном «рукаве», если оно имеет отношение к долгосрочному прогнозу в пределах каждого биологического подтипа РМЖ [6-8].

Существует два главных патоморфологических измерения резидуального заболевания резецированного препарата: урstage (American Joint Comission on Cancer Stage) и «оценка ложа остаточной опухоли (residual cancer burden- [RCB]). Метод RCB использует принципы «патоморфологических образцов-шаблонов» (pathologic sampling) и сообщений (отчетов), что также необходимо для точного определения наличия опухоли и ур-стадии любого резидуального заболевания после неоадъювантного лечения [9-12]. Следовательно, эта методика обеспечивает стандартизированную операционную процедуру для проспективной оценки постнеоадъювантных препаратов, требующую для исследования только стандартные гистологические препараты, минимальное время без дополнительных расходов [13-15]. Публичный web-сайт обеспечивает образовательные видеоматериалы для патологов, включая онлайн-калькулятор для определения шкалы индекса RCB (RCB index score) и класca RCB [16].

Шкала индекса RCB определяется из наиболее крупных зон и клеточности остаточной инвазивной первичной опухоли и числа метастатических лимфоузлов и размера наибольшего метастаза. Полный патоморфологический регресс (ответ) - pCR (стадияypT0/is, ypN0) эквивалентен $\mathrm{RCB}=0$; a $\mathrm{RCB}$ класс квалифицируется как минимальный (RCB-I), умеренный (средний) (RCB-II), экстенсивный (обширный) (RCB-III) на основании предопределенных «разделительных срезов» (cut points) 1,36 и 3,28 шкал индекса RCB [17-19].

В исследование Symmans было включено пять проспективных когорт пациентов с РМЖ с целью проверить долгосрочную прогностическую надежность измерения индекса и класса RCB после неоадъювантной химиотерапии в пределах каждого фенотипического подтипа РМЖ.

\section{Материалы и методы исследования}

Индекс RCB ассоциировался с риском рецидива или смерти, а пропорциональные риски (hazard ratios) в классах RCB были достоверны во всех пяти когор- тах, скорректированных по возрасту, клинической стадии и степени злокачественности. Индекс массы и диабетический статус не сказывались на прогнозе в любой когорте. Хотя изначально индекс RCB разрабатывался на данных когорты T/FAC-1, Symmans и соавт. наблюдали схожие долгосрочные прогностические результаты и в других когортах. RCB индекс и RCB классы добавляли независимую прогностическую информацию к ур стадии в рамках мультивариантных моделях и к ур-N стадии [20-22]. Кроме того, редкие рецидивы при достижении $\mathrm{pCR}$ не имели очевидной ассоциации с хирургической процедурой, радиографии препарата или с фенотипом опухоли [23-25].

\section{Трижды-негативный РМЖ: T/FАС химиотерапия}

Наблюдалась следующая пропорция пациентов в различных классах RCB: $35 \%$ pCR, $15 \%$ RCB-I, $33 \%$ RCB-II, $17 \%$ RCB-III. Хороший прогноз наблюдался у пациентов, достигших pCR (5-летняя безрецидивная выживаемость 94\%, 10-летняя RFS-86\%). В классе RCB-I - пятилетняя RCB достигала $89 \%$. 10 -летняя $-81 \%$. Прогноз ухудшался у пациентов c RCB-II (пятилетняя RFS-62\% и 10-летняя RFS$55 \%$ ). В классе RCB-III 5-летняя RFS равнялась лишь $26 \%$, а десятилетняя RFS-23\%. В мультивариантной модели для оценки безрецидивной выживаемости только индекс RCB имел независимое прогностическое значение ( HR 1.92), в то время как возраст, клинические стадия, степень злокачественности, мультифокальность и $\mathrm{pCR}$ не имели такого значения [26, 27].

\section{ER-позитивный/HER2-негативный РМЖ T/FAC химиотерапия}

В зависимости от RCB класса пропорция пациентов распределялись следующим образом: pCR$10 \%$, RCB-I $-13 \%$, RCB-II $-60 \%$ и RCB-III $-17 \%$. Наблюдался хороший прогноз у пациентов, достигших pCR (5-летняя безрецидивная выживаемость (RFS) равнялась $88 \%$, а десятилетняя - 83\%). При RCB-I 5-летняя безрецидивная выживаемость равнялись $100 \%$, а 10-летняя RFS равнялась $97 \%$ ). Пациенты с классом RCB-II характеризовались такой же 5-летней безрецидивной выживаемостью, что и при достижении $\mathrm{pCR}-87 \%$, но более низкой 10 -летней выживаемостью - 74\%. Однако обширное (экстенсивное) резидуальное заболевание (RCB-III) знаменовало значительное ухудшение прогноза: 5-летняя безрецидивная выживаемость $70 \%, 10$-летняя $52 \%$. При мультивариантном анализе индекс RCB, первичная стадия III и (парадоксально) pCR были независимыми прогностическими признаками, в то время как возраст, степень злокачественности и мультифокальность такими свойствами не обладали $[28,29]$.

HER2-позитивный РМЖ:

одна химиотерапия по схеме T/FAC

В зависимости от RCB класса пропорция больных распределилась следующим образом: pCR $37 \%$; RCB-I - $17 \%$, RCB-II - $31 \%$; RCB-III - $15 \%$. Показатели 5-летней безрецидивной выживаемости (RFS) оказались наиболее высокими при достижении pCR: RFS-94\% r 5-му году и $88 \%$ через 10 лет). 5-летняя выживаемость при классе RCB-I равнялись $89 \%$ и $63 \%$ через 10 лет. Долгосрочный прогноз для RCBII и RCB-III оказался схожим: 5-летняя RFS-62\%, 10 -летняя - $44 \%$ при RCB-II; 5-ти летняя и 10-летняя 
RFS для RCB-III - 47\%. В мультивариантной модели при оценке RFS оказались независимо значимыми вариабельностями следующие признаки: ядерная степень злокачественности, мультифокальность. А возраст, клиническая стадия и индекс RCB таким свойством не обладали.

HER2-позитивный РМЖ:

химиотерапия T/FEC с трастузумабом

В зависимости от класса RCB пропорция пациентов распределилась следующим образом: pCR $45 \%$, RCB-I - $19 \%$, RCB-II - $29 \%$ и RCB-III - $7 \%$. Классы RCB имели прогностическое значение в подгруппах с ER-/HER2+ и ER+/HER2-опухолями. Пациенты, достигшие $\mathrm{pCR}$ имели превосходный долгосрочный прогноз (5-летняя и 10-летняя RFS-95\%) что значительно выше, чем при других классах RCB. 5-летняя RFS при RCB-I равнялась $81 \%$, а 10-летняя $-77 \%$. При классе RCB-III 5-летняя и 10-летняя равнялись лишь $21 \%$. В мультивариантном анализе только индекс RCB или независимое прогностическое значение, в то время как возраст, исходная клиническая стадия, мультифокальность и даже pCR этим свойством не обладали.

\section{Результаты исследования и их обсуждение}

Индекс и классы RCB оказались прогностически значимыми при всех вариантах неоадъювантного лечения и всех фенотипических подтипах [30]. При фенотипах высокого риска - трижды негативном РМЖ (получавших схему T/FAC) и HER2-позитивном заболевании (получавших трастузумаб+T/FEC), индекс RCB был единственным прогностическим признаком в мультивариантной модели, включавшей оценку возраста, первоначальной клинической стадии (cTNM), степени злокачественности, мультифокальности и pCR [9]. При ER-позитивном/ HER2-негативном фенотипе индекс RCB и первоначальная сTNM имели независимое прогностическое значение.

$\mathrm{RCB}$ и ур-стадия вместе определяют величину резидуального заболевания в молочной железе и регионарных лимфоузлах, но определяют размер различно [13-15]. Индекс и класс RCB резидуального заболевания обладают независимым прогностическим свойством в мультивариантном анализе, включающем ур-стадию, ур-T, ур-N. RCBклассы усиливают прогностическую пригодность ур-стадии II и ур-стадии III, добавляя информацию к этим стандартным оценкам, свидетельствуя что оба фактора-RCB и урстадия (урT и урN) должны регистрироваться при резидуальном заболевании.

В отличие от системы CPS+EG (включающей клиническую стадию сTNM, урстадию, рецепторы эстрогенов и степень злокачественности), RCB не охватывает эту дополнительную долечебную информацию [19-21]. Кроме того, Neo-Bioscore, являющаяся модификацией CPS-EG (дополняется HER2-статус [23-25], являющаяся прогностической при РМЖ и способная усилить ур-стадию как прогностический инструмент, особенно при ER-позитивном/ HER2-негативном подтипе [26]. Однако $\mathrm{RCB}$ характеризует прогноз в пределах каждого фенотипического подтипа, подвергающихся различному лечению. Поэтому будущее усовершенствование RCB должно быть фенотипически специфичным, включая ребалансирование индекса RCB и разделительных числовых срезов (cut points) и подходящую комбинацию с долечебной (первичной) и биомаркерной информацией. При трижды-негативном РМЖ (ТНРМЖ) ответ на неоадъювантную химиотерапию был наиболее важной детерминантой выживаемости. Приблизительно половина ТНРМЖ популяции достигла pCR или RCB-I c хорошим прогнозом. Прогноз был хуже при RCB-II и RCB-III.

При ER+/HER2- РМЖ патоморфологический ответ на химиотерапию (RCB индекс) имел прогностическое значение, как и при других фенотипических подтипах. Поэтому, хотя дихотомное (dichotomous) отличие между $\mathrm{pCR}$ и резидуальным заболеванием представляется субоптимальным прогностическим суррогатом для ER+/HER- PMЖ [27], сама распространенность RCB является прогностически значимой. Более того резидуальный риск для пациентов с RCB-II и RCB-III повышается декадой позже: значит недостаточный ответ на химиотерапию может иметь отдаленные прогностические последствия для этих пациентов (несмотря на адъювантную эндокринотерапию).

Группа пациентов с RCB-II представляет важный вызов для улучшения прогностической стратификации, так как она охватывает приблизительно $60 \% \mathrm{ER}+/$ HER2-PMЖ и характеризуется $74 \%$ десятилетней безрецидивной выживаемостью (RFS). Резидуальный ER+/HER2-PMЖ имеет бимодальное распределение RCB и явно зависит от ур-N-статуса [17-19]. Тем не менее RCB оставалось прогностически значимым при позитивных (метастатических) лимфоузлах (урN+).

Более эффективная неоадъювантная химиотерапия может улучшить выживаемость ER+/HER2-заболевания, даже если частота pCR не изменяется, т.е. безотносительно pCR $[27,28]$. Точно так же меньшая масса (burden) резидуального заболевания совпадает с большей вероятностью пользы от адъювантной эндокринотерапии [30, 31]. Таким образом, распределение индекca RCB может оказаться информативным в рандомизированных клинических испыта- 
ниях, как бы то ни было, у пациентов с ER+/ HER2-заболеванием и RCB II наблюдается комплекс прогностических взаимосвязей, включающих распространение опухоли при первичном диагнозе, природную биологию, чувствительность к химиотерапии и чувствительность к адъювантной эндокринотерапии [22]. Понимание этой сложной системы может привести к излечению большего числа пациентов с II-III стадиями РМЖ.

При HER2-позитивном заболевании индекс RCB был прогностически значимым после одной химиотерапии и в ее комбинации с трастузумабом. Кроме того наблюдался прекрасный 10 -летний прогноз для $\mathrm{pCR}$ в когорте, получавшей трастузумаб+химиотерапию (H+T/FEC), вызывая размышление о полном излечении с помощью последовательного применения трастузумаба и химиотерапии, послеоперационного (адъювантного) трастузумаба и возможного иммунологического «надзора» (surveillance). Это поддерживает критерий pCR как важная суррогатная «конечная точка» (end point) для клинических испытаний химиотерапии при HER2-позитивном PMЖ. RCB-I при ER+/HER2+ PMЖ прогностически уступает значимости $\mathrm{pCR}$, то же относится и к RCB-II.

Патоморфологический ответ в лимфатических узлах является связным прогностическим фактором, поддерживая использование биопсии сигнальных лимфоузлов после неоадъювантного лечения [32]. В исследовании ACOSOG Z1071 частота ложнонегативных заключений в «послелечебной» биопсии сигнальных лимфоузлов достигала $12,6 \%$ у пациентов с документированными метастатическими лимфоузлами до лечения [33]. Но дополнительная радиологическая локализация доказанных метастатических узлов (до лечения) улучшает их поиск снижая частоту ложно-отрицательных заключений до $2 \%$ и ниже, усиливая точность оценки RCB и ур-N стадию [34, 35].

\section{Заключение}

В целом индекс RCB является значимым прогностическим параметром для общей и безрецидивной выживаемости после неоадъювантной системной терапии во всех биологических подтипах РМЖ и в планировании назначения адъювантного лечения резидуального РМЖ.

\section{Список литературы}

1. Семиглазов В.Ф. и др. Неоадъювантная системная терапия рака молочной железы (ретроспективный и проспективный анализ) // Вопросы онкологии. - 2017. - Т. 63, № 2. - C. 256-260.

2. Семиглазов В.Ф. и др. Новые возможности лечения рака молочной железы: ингибиторы микротрубочек // Фарматека. - 2017. - № 17. - С. 45-49.
3. Symmans F., Wei C., Gould Rebekah et. al. Long-term prognostic risk after neoadjuvant chemotherapy associated with residual cancer burden and breast cancer subtype. J. Clin Oncol, 2017, vol. 35, № 10, pp. 1049-1060.

4. Cortazar P., Zhang L., Untch M., et al: Pathological complete response and long-term clinical benefit in breast cancer: The CTNeoBC pooled analysis. Lancet, 2014, vol. 384, pp. 164-172.

5. Food and Drug Administration, HHS: Pathologic complete response in neoadjuvant treatment of highrisk early-stage breast cancer: Use as an endpoint to support accelerated approval; guidance for industry; availability. Fed Regist, 2014, vol. 79, pp. 60476-60477.

6. Gianni L., Eiermann W., Semiglazov V., et al: Neoadjuvant and adjuvant trastuzumab in patients with HER2-positive locally advanced breast cancer (NOAH): Follow-up of a randomised controlled superiority trial with a parallel HER2-negative cohort. Lancet Oncol, 2014, vol. 15, pp. 640-647.

7. Berry D.A., Hudis C.A. Neoadjuvant therapy in breast cancer as a basis for drug approval. JAMA Oncol, 2015, vol. 1, pp. 875-876.

8. Hatzis C., Symmans W.F., Zhang Y., et al: Relationship between complete pathologic response to neoadjuvant chemotherapy and survival in triplenegative breast cancer. ClinCancerRes 2016, vol. 22, pp. 26-33.

9. DeMichele A., Yee D., Paoloni M., et al: Neoadjuvant as future for drug development in breast cancer-Response. Clin Cancer Res, 2016, vol. 22, pp. 269.

10. Symmans W.F., Peintinger F., Hatzis C., et al: Measurement of residual breast cancer burden to predict survival after neoadjuvant chemotherapy. J. Clin Oncol, 2007, vol. 25, pp. 4414-4422.

11. Bossuyt V., Provenzano E., Symmans W.F., et al: Recommendations for standardized pathological characterization of residual disease for neoadjuvant clinical trials of breast cancer by the BIGNABCG collaboration. Ann Oncol, 2015, vol. 26, pp. $1280-1291$.

12. Provenzano E., Bossuyt V., Viale G., et al: Standardization of pathologic evaluation and reporting of postneoadjuvant specimens in clinical trials of breast cancer: Recommendations from an international working group. Mod Pathol, 2015,vol. 28, pp. 1185-1201.

13. The University of Texas,MDAnderson Cancer Center Residual Cancer Burden Calculator. URL: www. mdanderson. org/breastcancer_RCB.

14. Hudis C.A., Barlow W.E., Costantino J.P., et al: Proposal for standardized definitions for efficacy end points in adjuvant breast cancer trials: The STEEP system. J. Clin Oncol, 2007, vol. 25, pp. 2127-2132.

15. Pocock S.J., Clayton T.C., Altman D.G. Survival plots of time-to-event outcomes in clinical trials: Good practice and pitfalls. Lancet, 2002, vol. 359, pp. 1686-1689.

16. Harrell F.E. Jr. Regression modelling strategies: With applications to linear models, logistic regression, and survival analysis. Springer-Verlag, New York. 2001.

17. Hess K.R., Levin V.A. Getting more out of survival data by using the hazard function. Clin Cancer Res, 2014, vol. 20, pp. 1404-1409.

18. Peintinger F., Sinn B., Hatzis C., et al: Reproducibility of residual cancer burden for prognostic assessment of breast cancer after neoadjuvant chemotherapy. Mod. Pathol, 2015, vol. 28, pp. 913-920.

19. Naidoo K., Parham D.M., Pinder S.E. An audit of residual cancer burden reproducibility in a UK context. Histopathology, 2017, vol. 70, pp. 217-222.

20. Corben A.D., Abi-Raad R., Popa I., et al: Pathologic response and long-term follow-up in breast cancer patients treated with neoadjuvant chemotherapy: A comparison between classifications and their practical application. Arch Pathol Lab Med, 2013, vol. 137, pp. 1074-1082. 
21. Romero A., Garc'1a-S'aenz J.A., Fuentes-Ferrer M., et al: Correlation between response to neoadjuvant chemotherapy and survival in locally advanced breast cancer patients. Ann. Oncol, 2013, vol. 24, pp. 655-661.

22. Sheri A., Smith I.E., Johnston S.R., et al: Residual proliferative cancer burden to predict long-term outcome following neoadjuvant chemotherapy. Ann. Oncol, 2015, vol. 26, pp. 75-80.

23. Lee H.J., Park I.A., Song I.H., et al: Comparison of pathologic response evaluation systems after anthracycline with/without taxane-based neoadjuvant chemotherapy among different subtypes of breast cancers. PLoS One, 2015, vol. 10:e137885.

24. Mittendorf E.A., Jeruss J.S., Tucker S.L., et al: Validation of a novel staging system for diseasespecific survival in patients with breast cancer treated with neoadjuvant chemotherapy. J. Clin. Oncol, 2011, vol. 29, pp. 1956-1962.

25. Mittendorf E.A., Vila J., Tucker S.L., et al: The Neo-Bioscore update for staging breast cancer treated with neoadjuvan chemotherapy: Incorporation of prognostic biologic factors into staging after treatment. JAMA Oncol, 2016, vol. 2, pp. 929-936.

26. Marme' F., Lederer B., Blohmer J.U., et al: Utility of the CPS+EG staging system in hormone receptor-positive, human epidermal growth factor receptor 2-negative breast cancer treated with neoadjuvant chemotherapy. Eur. J. Cancer, 2016, vol. 53, pp. 65-74.

27. Von Minckwitz G., Untch M., Blohmer J.U., et al: Definition and impact of pathologic complete response on prognosis after neoadjuvant chemotherapy in various intrinsic breast cancer subtypes. J. Clin. Oncol, 2012, vol. 30, pp. 1796-1804.

28. Von Minckwitz G., Untch M., Nuesch E., et al: Impact of treatment characteristics on response of different breast can- cer phenotypes: Pooled analysis of the German neo-adjuvant chemotherapy trials. Breast Cancer Res Treat, 2011, vol. 125, pp. $145-156$.

29. Von Minckwitz G., Blohmer J.U., Costa S.D., et al: Response-guided neoadjuvant chemotherapy for breast cancer. J. Clin. Oncol, 2013, vol. 31, pp. 3623-3630.

30. Symmans W.F., Hatzis C., Sotiriou C., et al: Genomic index of sensitivity to endocrine therapy for breast cancer. J. Clin. Oncol, 2010, vol. 28, pp. 4111-4119.

31. Peintinger F., Buzdar A.U., Kuerer H.M., et al: Hormone receptor status and pathologic response of HER2-positive breast cancer treated with neoadjuvant chemotherapy and trastuzumab. Ann. Oncol, 2008, vol. 19, pp. 2020-2025.

32. Mougalian S.S., Hernandez M., Lei X., et al: Tenyear outcomes of patients with breast cancer with cytologically confirmed axillary lymph node metastases and pathologic complete response after primary systemic chemotherapy. JAMA Oncol, 2016, vol. 2, pp. 508-516.

33. Boughey J.C., Suman V.J., Mittendorf E.A., et al: Sentinel lymph node surgery after neoadjuvant chemotherapy in patients with node-positive breast cancer: The ACOSOG Z1071 (Alliance) clinical trial. JAMA 2013, vol. 310, pp. 1455-1461.

34. Caudle A.S., Yang W.T., Krishnamurthy S., et al: Improved axillary evaluation following neoadjuvant therapy for patients with node-positive breast cancer using selective evaluation of clipped nodes: Implementation of targeted axillary dissection. J Clin Oncol, 2016, vol. 34, pp. 1072-1078.

35. Grambsch P.M., Therneau T.M. Proportional hazards tests and diagnostics based on weightedresidual. Biometrika, 1994, vol. 81, pp. 515-526. 